\section{Uma Amostra de Pacientes com Diabetes Tipo I no Sul do Brasil}

\section{RESUMO}

Objetivos: Descrever uma população de pacientes com diabetes tipo 1 (DM1) em relação a fatores demográficos, ambientais, sócio-econômicos e manejo da doença. Delineamento: Série de casos. Participantes: Indivíduos com DMl, com até 10 anos de doença, até 30 anos de idade, residentes em onze municípios do sul do Brasil. Resultados: Foram estudados 126 indivíduos com DM1 (57 homens e 69 mulheres), sendo que a idade mais freqüente de início da doença foi dos 11 aos 15 anos (31\%). Houve variação sazonal na época de apresentação. O diagnóstico foi feito por sintomas que motivaram uma dosagem de glicemia em 61\%, por hospitalização, não em UTI, em 22\% e por cetoacidose em 18\%. Na amostra, 47\% aplicava insulina uma vez ao dia. Sessenta por cento dos pacientes realizava algum tipo de automonitorização, um terço reutilizava seis ou mais vezes a seringa e $50 \%$ da insulina era fornecida pelo poder público. Quanto ao reconhecimento da hipoglicemia, $18 \%$ dos pacientes não sabiam citar nenhum dos sinais de alerta. Grande parte da amostra (73\%) consultava médico especialista em DM. Dos pacientes com mais de 5 anos de doença, $16 \%$ nunca haviam feito fundoscopia e $17 \%$ haviam realizado o exame há 2 anos ou mais. As formas de apresentação da doença e da aquisição de insulina e a consulta com especialista estiveram associadas à renda familiar. As mulheres seguiam a dieta de modo mais adequado $(\mathrm{p}=$ 0,05) e auto-aplicavam insulina com mais freqüência, quando comparadas aos homens. Conclusões: Os fatores sócio-econômicos influenciaram neste estudo a forma de diagnóstico da doença, a obtenção de insulina e o acesso à especialistas. Os pacientes ainda carecem de conhecimentos básicos a respeito do manejo da doença. (Arq Bras Endocrinol Metab 2001;45/5:433-440)

Unitermos: Diabetes mellitus tipo 1; Epidemiologia; Fatores sócioeconômicos; Fatores demográficos.

\begin{abstract}
Objectives: To describe a type 1 diabetes mellitus (DM1) population with regard to demographic, socioeconomic, environmental and management aspects. Design: Series of cases. Participants: 126 DM1 patients, aged up to 30 yrs, with no more than 10 yrs of diagnosis, living in some cities of South Brazil. Results: The patients were 57 men and 69 women, being the age of onset mainly at 11-15 yrs (31\%). There were seasonal presentations at diagnosis. The diagnosis of DM was done as outpatients, by symptoms in $61 \%$, due to hospitalization in $22 \%$ and ketoacidosis in $18 \%$. Forty-seven percent of the sample injected insulin once daily, $60 \%$ of patients performed some self monitoring, one third reused the same syringe six or more times and $50 \%$ of insulin was provided by public services. Regarding awareness of hypoglycaemia, $18 \%$ of the patients did not know any signal of alert. A significant part of the sample was seen by a specialist in DM (73\%). Among the patients with five years or more of DM, $16 \%$ of them have never had their eyes examined and $17 \%$ had been examined at least two years ago. The onset of DM, assistance by a specialist and provision of insulin was associated with familiar income. Women were on diet more often $(p=0,05)$ and had more self-injection of insulin $(p=0,01)$ than men. Conclusions: In this
\end{abstract}

\section{artigo original}

\author{
Vera Maria Freitas da Silveiva \\ Ana Maria Baptista Menezes \\ Cora Luiza Araújo Post \\ Eduardo Coelbo Machado
}
Departamentos de Clínica Médica (VMFS, $A M B M, E C M)$ e de Nutrição (CLAP), Faculdade de Medicina, Universidade Federal de Pelotas, RS


study, socio-economic factors seem to influence the onset of DM, provision of insulin and assistance by a specialist doctor in DM. In general, patients did not have background and skills for self-management of DM. (Arq Bras Endocrinol Metab 2001;45/5:433-440)

Keywords: Type 1 diabetes mellitus; Epidemiology; Socioeconomic factors; Demographic factors.

$\mathrm{O}$ DIABETES MELLITUS TIPO 1 (DMl) é o distúrbio endócrino-metabólico crônico mais freqüente na infância (1). Apesar de poder ocorrer em qualquer idade, costuma manifestar-se abaixo dos trinta anos, concentrando-se no período escolar e na adolescência (2-4).

No Brasil foram publicados dois estudos que avaliaram a incidência de DMl. Em Londrina, a taxa encontrada foi de 12,7/100.000 (5), enquanto que em quatro cidades do estado de São Paulo a incidência de $\mathrm{DMl}$, em jovens abaixo de 15 anos, foi de 7,6/100.000 (6). A incidência mundial varia muito, com as mais baixas incidências no Continente Asiático $(0,1 / 100.000)$, e as mais altas na Finlândia e Sardenha $(36,5 / 100.000)(7)$.

As características demográficas dos pacientes com DMl são semelhantes em todo mundo e a incidência distribui-se igualmente entre os sexos $(2,8,9)$.

A classe social mais afetada no DMl é um dado controverso na literatura, alguns estudos mostrando uma maior incidência nas classes de renda mais alta (10-12) e outros, nas de renda mais baixa $(13,14)$.

Quanto à sazonalidade na apresentação da doença, tem sido descrito predomínio nos meses de inverno $(3,9,14-16)$.

Estudos descritivos sobre populações de indivíduos com DM são importantes para comparar as características dos pacientes com o restante da população e com diabéticos de outros países. Este conhecimento permite planejar e instituir políticas de saúde para uma adequada prevenção e controle da doença. Assim sendo, o objetivo do presente estudo foi descrever o perfil de uma amostra de pacientes com DMl, atendidos em Pelotas e alguns municípios vizinhos.

\section{PACIENTES E MÉTODOS}

Um prévio estudo caso-controle de pacientes com DMl foi realizado de janeiro de 1996 a junho de 1997, em alguns municípios da região sul do Brasil. Os casos foram pacientes com DMl, definidos por quadro de cetoacidose inicial ou necessidade de uso de insulina desde o diagnóstico, com idade entre 0 e 30 anos e, no máximo, 10 anos de diagnóstico, residentes em Pelotas e outros dez municípios vizinhos (Rio Grande, Arroio Grande, Jaguarão, Bagé, Canguçu, Piratini, Pedro Osório, Santa Vitória do Palmar, São Lourenço do Sul e Camaquã). Cabe ressaltar que a região estudada caracteriza-se por ser de economia agropecuária, com alguns dos municípios concentrando a maior parte de sua população na zona rural. Pelotas é a maior cidade da região (com cerca de 300.000 habitantes), concentrando o atendimento médico especializado.

A lista dos pacientes foi obtida a partir de contatos com todos os endocrinologistas de Pelotas, Rio Grande, Jaguarão e outras cidades da região onde trabalhavam endocrinologistas. Nas outras, foram contatados pediatras e clínicos diabetólogos. Também foi obtida uma relação de pacientes com DM atendidos nos postos da rede municipal de saúde de Pelotas, Unidades de Terapia Intensiva pediátricas e de adultos e daqueles que retiravam insulina no Centro de Saúde local ou participavam da Associação de Diabéticos de Pelotas e Rio Grande, cidades onde existiam Associações de Diabéticos.

Dos 167 pacientes listados destas várias fontes que potencialmente poderiam ser os casos, oito não foram localizados por falta de endereço ou endereço insuficiente, três já haviam falecido e 30 foram excluídos por não preencherem os critérios estabelecidos para este estudo. O presente artigo mostra a análise descritiva dos 126 pacientes restantes.

A coleta de dados foi realizada junto aos pacientes e/ou suas mães, através de um questionário padrão, aplicado por entrevistador em visita domiciliar. Também se utilizou um questionário específico aplicado pela médica coordenadora da pesquisa. Foram coletadas variáveis demográficas, sócio-econômicas e variáveis relativas à doença e seu controle, tais como idade de início, forma de apresentação, monitorização, manejo do DM e eventos estressantes ocorridos no ano anterior ao diagnóstico.

Foram feitas duas digitações independentes no Epi-Info 6.02. Para análise uni e bivariada foi utilizado o SPSS 6.1.

\section{RESULTADOS}

A tabela 1 mostra as características demográficas e sócio-econômicas dos pacientes com DM estudados. A população foi, em sua grande maioria, branca $(90,5 \%)$, com discreto predomínio feminino (55\%). A faixa etária de 11 a 15 anos correspondeu à maior parte da amostra (31\%). A média de idade nas mulheres foi de 
Tabela 1. Distribuição das variáveis demográficas e sócioeconômicas de 126 pacientes diabéticos tipol.

\begin{tabular}{|c|c|c|}
\hline \multicolumn{3}{|c|}{ Região Sul, RS, 1996-1997 } \\
\hline CARACTERISTICAS & $\mathrm{N}$ & $\%$ \\
\hline \multicolumn{3}{|l|}{ Sexo } \\
\hline Masculino & 57 & $45,2 \%$ \\
\hline Feminino & 69 & $54,8 \%$ \\
\hline \multicolumn{3}{|l|}{ Cor } \\
\hline Branca & 114 & $90,5 \%$ \\
\hline Não branca & 12 & $9,5 \%$ \\
\hline \multicolumn{3}{|l|}{ Idade } \\
\hline Até 10 anos & 23 & $18,3 \%$ \\
\hline 11-15 anos & 39 & $31,0 \%$ \\
\hline $16-20$ anos & 32 & $25,4 \%$ \\
\hline $21-30$ anos & 32 & $25,4 \%$ \\
\hline \multicolumn{3}{|c|}{ Renda familiar em salários mínimos } \\
\hline Até $3 \mathrm{sm}$ & 46 & 36,5 \\
\hline$>3 \mathrm{sm}$ & 80 & 63,5 \\
\hline \multicolumn{3}{|l|}{ Escolaridade $(n=117)^{*}$} \\
\hline Sem escolaridade & 1 & $0,9 \%$ \\
\hline $1-4$ anos & 25 & $21,4 \%$ \\
\hline 5-8 anos & 54 & $46,2 \%$ \\
\hline$=9$ anos & 37 & $31,6 \%$ \\
\hline
\end{tabular}

$17,2 \pm 6,8$ e, nos homens, $15,4 \pm 6,4$ anos. Em relação à renda familiar, apenas $36,5 \%$ tinham renda até 3 salários mínimos e $63,5 \%$ acima de 3 salários mínimos $(\mathrm{p}=0,004)$.

Os pacientes apresentaram escolaridade média de 6,6 anos, incluindo apenas aqueles com idade igual ou superior a 7 anos, quando tradicionalmente ocorre o ingresso escolar. Apenas uma paciente de 19 anos não foi à escola, por deficiência mental. Em relação à

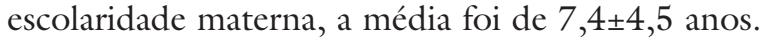

As famílias em estudo residiam predominantemente na zona urbana, sendo que $35 \%$ dos pacientes moravam ou já haviam morado na zona rural.

$\mathrm{Na}$ tabela 2 podem ser observadas algumas características da doença. A idade de início concentrouse dos 6 aos 15 anos, com idade média de $11,3 \pm 6,1$ anos. O período de apresentação mais freqüente foi de abril a julho e dentre as formas de apresentação da doença, a presença de sinais e sintomas sugestivos de DM foi a mais freqüente $(61 \%)$, seguida pela internação hospitalar por hiperglicemia $(22 \%)$ e internação em UTI por cetoacidose diabética (18\%).

Em relação à história familiar de $\mathrm{DM}$ em parentes de primeiro grau, 23 pacientes (18\%) relataram
Tabela 2. Características de apresentação do diabetes mellitus tipo 1 (DM1). Região Sul, RS, 1996, 1997

\begin{tabular}{lcc}
\hline CARACTERÍsTICAS & $\mathrm{N}$ & $\%$ \\
\hline ldade de início do DM $(n=126)$ & & \\
1-5 anos & 25 & $19,8 \%$ \\
6-10 anos & 33 & $26,2 \%$ \\
$11-15$ anos & 39 & $31,0 \%$ \\
$16-20$ anos & 19 & $15,1 \%$ \\
$\geq 21$ anos & 10 & $7,9 \%$ \\
& & \\
Época de início do DM $(n=120)$ & 34 & $28,3 \%$ \\
dezembro a março & 49 & $40,8 \%$ \\
abril a julho & 37 & $30,8 \%$ \\
agosto a novembro & & \\
Forma de apresentação $(n=125)$ & 76 & $60,8 \%$ \\
sinais e sintomas & & \\
internação por hiperglicemia & 27 & $21,6 \%$ \\
coma & 22 & $17,6 \%$ \\
\hline
\end{tabular}

familiar diabético (não tendo sido caracterizado o tipo de DM). Cerca de metade deles referiu o pai como o familiar afetado (48\%), seguido dos irmãos (30\%), pai e/ou mãe + irmão (13\%) e mãe (9\%).

Quanto ao uso de insulina, a maioria dos pacientes auto-aplicava a insulina $(71 \%)$, sendo que num segundo grupo era a mãe que, mais freqüentemente, realizava a aplicação. Os pacientes iniciaram a auto-aplicação de insulina ainda jovens: $24 \%$ dos casos aplicou insulina com 10 anos de idade ou menos (um destes com 6 anos) e 40\% iniciou a aplicação entre 11 e 15 anos. Cerca de metade dos pacientes fazia uso apenas de uma aplicação matinal de insulina NPH. A insulina regular era utilizada regularmente por cerca de $20 \%$ dos pacientes. Metade dos pacientes obtinha insulina no sistema público de saúde. Quanto ao local de armazenamento, a maior parte dos pacientes guardava a insulina em uso na geladeira $(85,5 \%)$. Mais de $60 \%$ dos pacientes reutilizavam seringas de 2 a 5 vezes e $32 \%$ reutilizavam seis vezes ou mais.

Quanto à monitorização do controle do DM, $61 \%$ dos entrevistados diziam realizar testes de automonitorização, sendo que destes apenas $27 \%$ realizavam o teste antes das principais refeições. Em $73 \%$ dos casos o teste era realizado no sangue e em $27 \%$, na urina.

$\mathrm{Na}$ tabela 3 estão as variáveis relacionadas aos sinais e sintomas de hipoglicemia e seu reconhecimento. Da amostra estudada $18 \%$ dos pacientes não sabia citar nenhum sintoma de hipoglicemia. Os sintomas mais freqüentes, segundo a percepção dos pacientes, foram tremores $(51 \%)$, tonturas $(47 \%)$, cansaço ou fraqueza $(38 \%)$ e sudorese $(35 \%)$, seguidos de alteração de consciência, distúrbios visuais, fome e outros. 
Tabela 3. Variáveis relacionadas a hipoglicemia em pacientes com diabetes tipo 1. Região Sul, RS, 1996-97.

\begin{tabular}{lcr}
\hline VARIÁVEIS & $\mathrm{N}$ & $\%$ \\
\hline Sintomas de hipoglicemia $(n=121)$ & & \\
$\quad$ não reconhece & 22 & $18,2 \%$ \\
refere um sintoma & 5 & $4,1 \%$ \\
refere dois sintomas & 39 & $32,2 \%$ \\
refere três sintomas & 41 & $33,9 \%$ \\
refere quatro sintomas e mais & 14 & $11,6 \%$ \\
& & \\
Freqüência de sintomas $(n=99)^{*}$ & & \\
tremor & 50 & $50,5 \%$ \\
tonturas & 46 & $46,5 \%$ \\
cansaço/fraqueza & 38 & $38,4 \%$ \\
sudorese & 35 & $35,4 \%$ \\
alteração de consciência & 19 & $19,2 \%$ \\
distúrbios visuais & 17 & $17,2 \%$ \\
fome & 14 & $14,1 \%$ \\
outros sintomas & 44 & $44,4 \%$ \\
\hline
\end{tabular}

* O total excede $100 \%$, pois um paciente pode referir 0 reconhecimento de até 5 sintomas.

A maior parte dos pacientes estudados (73\%) informou que consultava médico especialista em DM. Dos 63 pacientes com 5 ou mais anos de DM, 67\% haviam feito o exame de fundo de olho no ano ante- rior, $17 \%$ há dois anos ou mais e $16 \%$ nunca haviam realizado fundoscopia.

Em relação à dieta para $\mathrm{DM}, 23 \%$ relataram não fazê-la, $2 \%$ faziam-na às vezes, enquanto que $74 \%$ da amostra referia fazer dieta regularmente.

$\mathrm{Na}$ tabela 4 está descrita a análise bivariada das variáveis relativas ao manejo da doença conforme renda familiar dicotomizada em menor ou igual a 3 salários mínimos e maior de 3 salários mínimos. A forma de apresentação do DM esteve significativamente associada à renda familiar: $74 \%$ dos pacientes de maior renda tiveram diagnóstico feito através de exames laboratoriais a partir de sintomas sugestivos de $\mathrm{DM}$, enquanto que quase dois terços dos pacientes com menor renda tiveram diagnóstico durante internação hospitalar.

O local de consultas esteve associado à renda, já que $76 \%$ dos pacientes com renda familiar superior a 3 salários mínimos eram atendidos em consultório particular, enquanto que $44 \%$ dos de renda familiar inferior recebiam atendimento em local semelhante.

Consulta com médico especialista também mostrou associação significante com relação à renda $(\mathrm{p}=0,03)$, sendo que aqueles com renda superior a 3

Tabela 4. Distribuição dos pacientes com diabetes tipo $1(n=126)$ segundo renda familiar. Região Sul, RS, 1996-97.

\begin{tabular}{|c|c|c|c|}
\hline \multirow[t]{2}{*}{ VARIÁVEIS } & \multicolumn{2}{|c|}{ RENDA FAMILIAR } & \multirow[t]{2}{*}{ SIGNIFICÂNCIA } \\
\hline & $\leq 3 \mathrm{SM}$ & $>3 \mathrm{SM}$ & \\
\hline $\begin{array}{l}\text { Forma de apresentação } \\
\text { exames laboratoriais } \\
\text { internação } \\
\text { coma }\end{array}$ & $\begin{array}{l}17(38 \%) \\
14(31 \%) \\
14(31 \%)\end{array}$ & $\begin{array}{l}59(74 \%) \\
13(16 \%) \\
8(10 \%)\end{array}$ & $p<0,001$ \\
\hline $\begin{array}{l}\text { Local de consulta } \\
\text { particular } \\
\text { ambulatório de ensino/outros } \\
\text { postos ou centros de saúde }\end{array}$ & $\begin{array}{l}20(44 \%) \\
9(20 \%) \\
16(36 \%)\end{array}$ & $\begin{array}{c}60(76 \%) \\
12(15 \%) \\
7(9 \%)\end{array}$ & $p<0,001$ \\
\hline $\begin{array}{l}\text { Médico especialista } \\
\text { sim } \\
\text { não }\end{array}$ & $\begin{array}{l}27(60 \%) \\
18(40 \%)\end{array}$ & $\begin{array}{l}62 \text { (79\%) } \\
17(22 \%)\end{array}$ & $p=0,03$ \\
\hline $\begin{array}{l}\text { Sintomas de hipoglicemia } \\
\geq \text { quatro sintomas } \\
\text { três sintomas } \\
\text { dois sintomas } \\
\leq \text { um sintoma }\end{array}$ & $\begin{array}{l}5(11 \%) \\
14(32 \%) \\
8(18 \%) \\
17(39 \%)\end{array}$ & $\begin{array}{l}9(12 \%) \\
27(35 \%) \\
31(40 \%) \\
10(13 \%)\end{array}$ & $p=0,006$ \\
\hline $\begin{array}{l}\text { Fundo de olho* } \\
\leq \text { um ano } \\
\geq \text { dois anos } \\
\text { nunca fez }\end{array}$ & $\begin{array}{l}18(40 \%) \\
8(18 \%) \\
19(42 \%)\end{array}$ & $\begin{array}{l}41(52 \%) \\
8(10 \%) \\
30(38 \%)\end{array}$ & $p=0,32$ \\
\hline
\end{tabular}

* Refere-se apenas a pacientes com 5 anos ou mais, de diagnóstico de diabetes $(n=63)$. $\mathrm{SM}=$ Salário mínimo 
salários mínimos consultavam mais especialistas do que os de menor renda ( $79 \%$ e $60 \%$, respectivamente).

$\mathrm{O}$ número de sintomas de hipoglicemia reconhecido pelos pacientes também esteve associado à renda $(p=0,006)$. Os pacientes com renda mais elevada apresentaram reconhecimento de sintomas de hipoglicemia levemente superior àqueles com renda mais baixa.

A compra de insulina esteve positivamente associada à renda familiar: $64 \%$ dos pacientes de maior renda compravam insulina, comparados com $27 \%$ daqueles de menor renda familiar $(\mathrm{p}<0,001)$.

As variáveis: exame de fundo de olho, número de consultas e de exames no último ano, realização de testes de automonitorização, reutilização de seringas e dieta não mostraram associação significante com renda familiar.

A mesma análise bivariada sobre o manejo da doença conforme sexo mostrou que as mulheres faziam mais dieta $(55,3 \% / 44,7 \% ; \mathrm{p}=0,05)$ e auto-aplicavam a insulina mais do que os homens $(\mathrm{p}=0,01)$. As demais variáveis não mostraram associação significante em relação ao sexo.

Consulta com médico especialista mostrou-se positivamente associada ao maior reconhecimento dos sintomas de hipoglicemia $(\mathrm{p}=0,02)$, maior uso de insulina regular matinal $(\mathrm{p}=0,006)$ e de testes de automonitorização $(\mathrm{p}=0,004)$; controlando para renda familiar estas variáveis permaneceram significantes apenas nas faixas de menor renda.

\section{DISCUSSÃO}

Os estudos descritivos são de grande valor para estabelecer o perfil dos pacientes com DM e definir metas a serem traçadas quanto ao manejo da doença. A partir de seus resultados algumas medidas podem ser recomendadas e adotadas pelos Serviços e Políticas de Saúde.

A amostra estudada foi constituída por casos de DM selecionados a partir de vários locais de atendimento em Pelotas. Acredita-se que a grande maioria dos pacientes com DMl em Pelotas foi captada pelo sistema de busca utilizado, uma vez que essa doença requer algum acompanhamento médico, embora não tenha sido realizado teste de captura/recaptura, que poderia mostrar melhor a cobertura.

Dos pacientes oriundos de outros municípios que não Pelotas (10\% em Rio Grande, 9,5\% Canguçu, 7\% Jaguarão, 4\% Santa Vitória do Palmar, 2\% São Lourenço do Sul e outras cidades com menos de 1\% cada), foram entrevistados apenas aqueles que consul- taram ou estiveram internados em Pelotas e/ou consultaram com especialista ou pediatras naquelas cidades. Isso poderia ter induzido algum tipo de viés, selecionando apenas os de maior renda; entretanto, acredita-se que pela gravidade da doença é provável que os pacientes com DMl façam pelo menos uma avaliação com especialista, especialmente nos primeiros anos após o diagnóstico. Todas as análises foram refeitas utilizando-se apenas os pacientes moradores de Pelotas, sem que houvesse diferenças nos resultados aqui apresentados.

O pequeno número de perdas devido a endereços incompletos, além das características acima descritas, sugere satisfatória representatividade da amostra para a área geográfica em questão.

A amostra estudada, embora não seja baseada em casos incidentes, não difere da literatura em termos de sexo, cor e da idade de início do DM $(2-4,8,9,17)$.

Quanto à distribuição da renda familiar, observou-se semelhança entre a amostra estudada e a de outros estudos de base populacional realizados no município de Pelotas: estudo de Olinto e col. (18) com mulheres em idade reprodutiva e o de Lima (19) com trabalhadores com carteira assinada (19) mostram a mesma distribuição de renda.

A sazonalidade, com predomínio do diagnóstico nos meses de inverno, não teve significância estatística nesta amostra. Tem sido postulado que as infecções, ocorridas especialmente nos meses de inverno, possam ter um papel desencadeante do processo auto-imune ou serem um fator de descompensação da doença (20). No Brasil, Campos e cols. (5) não observaram variação sazonal na incidência de diabetes em Londrina, PR.

Apesar de haver suspeita do diagnóstico através da manifestação de sintomas clínicos de diabetes em $61 \%$ dos casos, é importante destacar que a internação hospitalar por cetoacidose diabética (CAD) ocorreu em $18 \%$ dos pacientes, com todos os riscos de morbimortalidade a ela associados. Ainda cabe lembrar que esta última forma de apresentação esteve associada a fatores sócio-econômicos, ou seja, pacientes mais pobres foram mais hospitalizados. Ramos e cols. (17), em estudo no nordeste do Brasil, encontraram uma frequiência de $20 \%$ de CAD e $46 \%$ de internação hospitalar, ao diagnóstico do DM, na população diabética atendida em Campina Grande (Paraíba). Estudo de demanda, realizado no Rio de Janeiro, mostrou uma taxa de $31 \%$ de CAD (3).

Também foi encontrada associação entre hereditariedade e DMl, com presença mais freqüente de pai diabético, seguido pelo irmão, o que já foi demonstra- 
do na literatura $(21,22)$, embora em nossa população não tenhamos separado o tipo de DM dos familiares afetados, pela característica de nosso questionário. O perfil do DMl está bem estabelecido, com a presença de antígenos de superfície do sistema HLA que conferem maior susceptibilidade à doença (23) não tendo, no entanto, uma concordância maior do que $50 \%$ entre os gêmeos idênticos (24). Estudos como o projeto DIAMOND, da Organização Mundial de Saúde, estão em curso para promover análises dos potenciais riscos potenciais genéticos, ambientais e outros, nos parentes em primeiro grau dos pacientes com DMl (21).

Quanto à aplicação de insulina, um grande número de pacientes informou auto-aplicação (71\%), o que parece indicar uma preocupação na educação precoce do diabético.

Chama atenção que metade dos pacientes não usa uma segunda dose de insulina NPH em 24 horas, o que provavelmente dificulta um bom controle glicêmico. Em relação à insulina regular, apenas cerca de $20 \%$ dos pacientes utilizam-na regularmente no momento da pesquisa, o que contrasta com o DCCT (Diabetes Control and Complications Trial), que evidenciou que o bom controle do DM obtido por múltiplos testes e injeções diárias de insulina, evitava as complicações crônicas do DM (25).

A compra de insulina esteve associada com renda familiar, ou seja, $73 \%$ dos pacientes de menor renda retiravam a insulina no Centro de Saúde. Esse fato reforça a necessidade de acesso permanente a essa forma de obtenção de insulina.

$\mathrm{O}$ armazenamento da insulina em uso na geladeira pela quase totalidade dos pacientes pode sugerir um desconhecimento de que a insulina em uso pode ser guardada apenas em local seco e ao abrigo da luz, para evitar a sensação dolorosa do líquido gelado, conforme recomendação da Associação Americana de Diabetes (26). A conservação da insulina em uso no compartimento das verduras ou na porta da geladeira pode ser utilizada, devendo-se retorná-la à temperatura ambiente antes da aplicação. Mais de $90 \%$ dos pacientes reutilizavam as seringas, o que pode ser feito observando algumas regras de higiene tais como: lavar as mãos antes da aplicação, não manipular a agulha e guardar a seringa no próprio invólucro (27). Mais de um terço dos pacientes, no entanto, reutilizavam as seringas $6 \mathrm{ou}$ mais vezes, o que está acima do recomendado (26).

Embora $60 \%$ dos pacientes relatassem fazer testes de auto monitorização, apenas $27 \%$ dos mesmos realizavam rotineiramente teste antes das principais refeições, que é a monitorização mínima para tentar restaurar a normoglicemia $(25)$.
A orientação sobre o reconhecimento da hipoglicemia, principal complicação do uso da insulina, deve ser feita nos primeiros dias após o diagnóstico, bem como as recomendações de como evitá-la e tratá-la. Chama atenção que $18 \%$ da amostra não sabia citar nenhum sintoma de hipoglicemia. Em torno de $50 \%$ citava as tonturas, $19 \%$ as alterações de consciência e $17 \%$ os distúrbios visuais, todos sintomas de neuroglicopenia, o que já indica uma hipoglicemia mais grave.

$\mathrm{O}$ fato de consultar médico especializado em DM parece influenciar algumas variáveis que refletem o conhecimento e a educação do paciente para o bom manejo da doença: dos pacientes que relatavam fazer dieta, $82 \%$ consultaram especialista, valor este que permaneceu estatisticamente significativo, mesmo quando controlado para renda familiar. Não se pode afastar causalidade reversa, já que os pacientes auto-informavam a dieta, e poderiam estar mais conscientes da necessidade de fazê-la. Também um maior número de pacientes acompanhados por especialistas relatavam fazer testes de automonitorização, quando comparados àqueles que não consultaram, mesmo controlando para renda familiar.

É preocupante o fato de que $16 \%$ dos pacientes com mais de 5 anos de diagnóstico não haviam realizado nenhum exame de fundoscopia, especialmente após a recente análise de pacientes do DCCT, que demonstrou que $44,4 \%$ dos pacientes portadores de DMl de menos de 5 anos de duração já apresentavam evidências de retinopatia (28). O Wisconsin Epidemiologic Study Diabetic Retinopathy (WESDR) destaca que 37 a $50 \%$ dos pacientes com retinopatia diabética proliferativa não haviam sido submetidos a exame oftalmológico com fundoscopia nos últimos dois anos e $51 \%$ dos casos de alto risco não foram devidamente encaminhados para fotocoagulação (29). Também é referido na literatura o desconhecimento dos pacientes sobre a possibilidade de haver retinopatia sem queixas visuais (30).

Apesar da importância da alimentação adequada para o controle da doença, apenas $74 \%$ referiram seguir alguma dieta orientada, sendo que as mulheres mais do que os homens, o que pode ser devido à maior preocupação das mulheres com o peso. Embora se saiba que a dieta é parte importante do tratamento, sabe-se da dificuldade na mudança de hábitos alimentares, principalmente naqueles pacientes sem tratamento intensivo usando doses fixas de insulina de longa ação (31). Além de seguirem mais a orientação dietética, as mulheres também referiam mais autoaplicação de insulina do que os homens, sugerindo um maior cuidado com a doença. 
Como as informações sobre dieta, uso de várias doses de insulina e testes de monitorização, foram informadas pelos pacientes, estas podem não ser verdadeiras, especialmente naqueles pacientes mais orientados sobre a necessidade de controle. Como, em geral, tais cuidados ainda estiveram muito aquém do ideal, reforçam a qualidade inadequada de seu tratamento.

A educação do indivíduo com DM e de sua família, bem como a orientação aos profissionais que acompanham os pacientes com DMl, é essencial para o bom controle da doença, e auxilia na tentativa de evitar suas complicações crônicas. Isso reforça a necessidade de uma equipe multidisciplinar, incluindo profissionais especialistas em educação para orientar esses pacientes.

Apoiado em consensos internacionais (26) recomenda-se a implementação de algumas medidas que objetivam minimizar as complicações crônicas do DMl e reduzir a freqüência de hospitalizações:

1. Educação do paciente e familiares quanto ao reconhecimento dos sinais e sintomas de hiper e hipoglicemia, conhecimento das complicações crônicas do DM, conhecimento de testes para monitorização de glicemia e de quando usá-los, utilização de múltiplas doses de insulina ao dia, cuidados com a aplicação de insulina e com o uso de seringas, necessidade de consultas e exames periódicos e utilização de alimentação adequada;

2. Equipe multidisciplinar, composta por endocrinologista ou diabetologista, nutricionista, oftalmologista, enfermeiro, psicólogo e pedagogo;

3. Política de saúde voltada para a educação das populações mais carentes visando identificação dos sinais mais precoces de DM, com o fornecimento de insulina de forma mais regular, disponibilidade de equipe multidisciplinar nos serviços públicos de saúde e programas voltados para prevenção das complicações crônicas do DMl.

\section{REFERÊNCIAS}

1. WHO Diamond Project Group. WHO Multinational Project for Childhood Diabetes. Diabetes Care 1990;13: 1062-8.

2. Scott CR, Smith JM, Cradock MM, Pihoker C. Characteristics of youth-onset noninsulin-dependent diabetes mellitus and insulin-dependent diabetes mellitus at diagnosis. Pediatrics 1997; 100:84-91.

3. Cavalcante LF, Gonçalves MM, Santos AM, Aguiar LGK, Cunha EF, Gomes MB, et al. Aspectos clínicos e epidemiológicos dos pacientes com Diabetes Mellitus tipo 1 do ambulatório de Diabetes do HUPE-UERJ. $9^{\circ}$ Con- gresso da Associação Latino-Americana de Diabetes / $10^{\circ}$ Congresso Brasileiro de Diabetes (resumo). 1995.

4. Apud Gomes MB, Aguiar LGK, Marques EP, Sarno EN, Chacra AR. Estudo comparativo de variáveis epidemiológicas em casos múltiplos e esporádicos de Diabetes Mellitus do tipo 1. Arq Bras Endocrinol Metab 1996;40:13-5.

5. Campos JJB, Almeida HG, lochida LC, Franco LJ. Incidência de Diabetes Mellitus Insulino-Dependente ((Tipo 1) na cidade de Londrina, PR, Brasil. Arq Bras Endocrinol Metab 1998:42(1):36-44.

6. Ferreira SRG, Franco LJ, Vivolo MA, Negrato CA, Simões ACP, Venturelli CR. Population based incidence of IDDM in the state of São Paulo, Brazil. Diabetes Care 1993; 16:701-4.

7. Karvonen M, Viek-Kayander M, Moltchanova E, Libman I, Laporte R, Tuomilehto J, et al. For the Diamond Project. Incidence of childhood Type 1 Diabetes worldwide. Diabetes Care 2000;23(10): 1516-26.

8. Ferraro DM, Eandi ML, Ramos. Servicio de Nutricion y Diabetes Hospital Generalde Niños Pedro de Elizalde Buenos Aires, Argentina. Estudio Epidemiológico de la población de pacientes diabéticos del Servicio de Diabetes del Hospital General de Niños Pedro de Elizalde entre 1977-1994. $9^{\circ}$ Congresso da Associação LatinoAmericana de Diabetes / $10^{\circ}$ Congresso Brasileiro de Diabetes (resumo). 1995.

9. Michalková DM, Cernay JC, Danková A, Rusnák M, Fandáková K, Slovak. Childhood Diabetes Epidemiology Study Group. Incidence and prevalence of childhood diabetes in Slovakia (1985-1992). Diabetes Care $1995 ; 18: 315-20$.

10. Patterson C, Carson DJ, Hadden DR, Waugh NR, Cole SK. A case-control investigation of perinatal risk factors for childhood IDDM in Northern Ireland and Scotland. Diabetes Care 1994; 17:376-8.

11. Metcalfe MA, Baum JD. Family characteristics and insulindependent diabetes. Arch Dis Child 1992;67:731-6.

12. Khan N, Couper JJ. Low-birth-weight infants show earlier onset of IDDM. Diabetes Care 1994;17:653-6.

13. Blom L, Dahlquist $G$, Sandström A, Wall S. The Swedish childhood diabetes study-social and perinatal determinants for diabetes in childhood. Diabetologia 1989;32:7-13.

14. Larenas $G$, Manosalva $M$, Barthou $M$, Montecinos $A$, Vidal T. Departamento Medicina Interna, Facultad de Medicina, Universidad de la Frontera, Temuco, Chile. Estacionalidad y edad de comienzo de la diabetes mellitus isulino-dependiente en Ia IX Region-Chile. $9^{\circ}$ Congresso da Associação Latino-americana de Diabetes $/ 10^{\circ}$ Congresso Brasileiro de Diabetes. 1995.

15. Torres JMR, Rodrigo JAR. El Sindrome clinico de diabetes mellitus. In: Rull, Zorilla, Jadzinsky, Santiago. Diabetes Mellitus-Complicaciones crónicas. InteramericanaMcGraw-Hill; 1:3-16, 1992.

16. Samuelsson U, Jahansson C, Ludvigsson J. Breast-feeding seems to play a marginal role in the prevention of insulin-dependent diabetes mellitus. Diabetes Res Clin Pract 1993; 19:203-10

17. Ramos AJS, Araújo MM, Cavalcante GS, Tejo A, Vieira SAT, Bezerra CT, et al. Perfil do diabético Tipo I atendido em Campina Grande (PB). $9^{\circ}$ Congresso da Associação 
Latino-Americana de Diabetes $/ 10^{\circ}$ Congresso Brasileiro de Diabetes (resumo). 1995.

18. Olinto MT, Victora CG. Induced abortion in Brazil: Testing the Ballot-box Methodology. Rev Saúde Públ 1999; 33(1):64-72.

19. Lima R. Acidentes de trabalho em Pelotas: Um estudo de casos e controles. Dissertação apresentada à Faculdade de Medicina da Universidade Federal de Pelotas para obtenção de Título de Mestre em Epidemiologia, 1997.

20. Soltész $G$, Jeges S, Dahlquist $G$. Hungarian Childhood Diabetes Epidemiology Study Group. Non-genetic risk determinants for type 1 (insulin-dependent) diabetes mellitus in childhood. Acta Paediatr Scand 1994;83: 730-5.

21. The WHO Multinational Project for Childhood Diabetes Group. Familial insulin-dependent diabetes mellitus (IDDM) epidemiology: standardization of data for the DIAMOND project. Bull WHO 1991;69:767-77.

22. Rjasanowski I, Heink $P$, Michaelis D, Kurajewa TL. The high frequency of type I (insulin dependent) diabetes in fathers than in mothers of type l-diabetic children. Exp Clin Endocrinol 1990;95:91-6.

23. Wolf E, Spencer KM, Cudworth AG. The Genetic susceptibility of type-I (insulin-dependent) diabetes: analysis of the HLA-DR association. Diabetologia 1983;24:224-9.

24. Barnett AH, Eff C, Leslie RDG. Diabetes in identical twins. A study of 200 pairs. Diabetologia 1981;20:87-93.

25. The Diabetes Control and Complications Trial Research Group. The effect of intensive treatment of diabetes prevents development and progression of long-term complications of insulin-dependent diabetes mellitus. $\mathbf{N}$ Engl J Med 1993:329:977-86.

26. American Diabetes Association: Clinical Practice Recommendations. Diabetes Care 2000;22(Suppl) 1.

27. Scain S. Reutilização de seringa descartável para aplicação de insulina. Rev Hosp Clín (PA) 1985;5:181-5.

28. Malone J I, Morrison AD, Pavan PR, Cuthbertson DD. Prevalence and significance of Retinopaty in subjects with Type 1 Diabetes of less than 5 years' duration screened for the Diabetes Control and Complications Trial. Diabetes Care 2001;24(3):522-6.

29. Klein R, Klein B, Moss SE. Epidemiology of proliferative diabetic retinopathy. Diabetes Care 1992; 15:1875-90.

30. Vilela MAP, Giacomet AC. Retinopatia diabética: Atualização em epidemiologia, fisiopatologia e curso natural. Rev Assoc Med RS 1995;39:10-5.

31. Bantle JP. Recomendações atuais relativas ao tratamento com dieta para Diabetes Mellitus. Arq Bras Endocrinol Metab 1995;39:141-6.

\section{Endereço para correspondência:}

Vera Maria Freitas da Silveira

Rua Félix da Cunha 614, sala 303

96.010-000 Pelotas, RS 